\title{
CLUSTERING BASED LOCAL TONE MAPPING ALGORITHM FOR DISPLAYING HDR IMAGES ON LDR DEVICES
}

\author{
Taeuk Kang, Jeonghyun Lee ${ }^{1}$ and JechangJeong ${ }^{2}$ \\ ${ }^{1}$ Department of Electronic and Computer Engineering, \\ Hanyang University, Seoul, Korea \\ ${ }^{2}$ Department of Electronic Engineering, Hanyang University, Seoul, Korea
}

\begin{abstract}
In order to display HDR (High Dynamic Range) images with increased dynamic range on LDR (Low Dynamic Range) monitors, it is necessary to perform a tone mapping technique, which is a process of compressing a dynamic range of an image. Representative techniques are global tone mapping and local tone mapping. Though global tone mapping is simple to compute, it has low local contrast and loses details. Local tone mapping has high local contrast but it demands high computational complexity. In order to take advantage and to compensate of two techniques, we propose local tone mapping based on clustering. Clustering reduces the complexity of local tone mapping implementation by dividing images. In the local tone mapping process, the local adaptation is obtained by combining the cluster-level log mean and global log mean. Using local characteristics, that is local adaptation, based on clustering, the results has high local contrasts and local detail is improved. Experiment result shows that proposed algorithm has better performance than conventional algorithm.
\end{abstract}

\section{KEYWORDS}

High Dynamic Range(HDR) Imaging, Tone Mapping, k-means Clustering,

\section{INTRODUCTION}

The dynamic range means the ratio of the brightest and darkest values in an image. The human visual system(HVS) has wide dynamic range. A human can perceive luminance ranging from $10^{\wedge}-6$ to $10^{\wedge} 8(\mathrm{~cd} / \mathrm{m} 2)$. Consumer display device normally presents luminance up to $500(\mathrm{~cd} / \mathrm{m} 2)$ [1]. Since it has smaller dynamic range than HVS, it doesn't have the ability to represent bright and dark region simultaneously. To solve this problem, high dynamic range (HDR) imaging technique emerged.

The purpose of HDR imaging technique is to capture all natural luminance range. HDR images are acquired using modern high-quality imaging sensor or exposure fusion technique. HDR image contains luminance up to $10,000(\mathrm{~cd} / \mathrm{m} 2)$. Because an HDR image can represent a wide dynamic range, they can express dark and bright region similar to the human eye [1]. Becausethe dynamic range of the low dynamic range(LDR) device is smaller than the dynamic range of HDR image, the dynamic range must be compressed in order to display the HDR image on the LDR device. This process is called tone mapping.

David C. Wyld et al. (Eds) : CNDC, DPPR, AIAA, WiMNET, ACITY - 2017

pp. 41- 51, 2017. (C) CS \& IT-CSCP 2017

DOI : $10.5121 /$ csit.2017.71605 
Tone mapping is divided into a global tone mapping and a local tone mapping. Global tone mapping is a way to treat all pixels equally using a simple function when processing one image. Since this method handles all pixels in the same way, it has low computational complexity and is easy to design, but it does not take local characteristics into account and loses details corresponding to high frequencies. In addition, the local tone mapping is a method of calculating based on local characteristics when processing an image. This technique has high computational complexity because it considers regional characteristics, but has the advantage of implementing tone mapping while preserving the details of high frequencies. In this case, however, there is a high probability that defects such as a halo artifacts or gradient reversal[2].

In other classification, the tone mapping is divided into two parts, one based on HVS and the other constructed through experiments. As an example, tone mapping algorithm of Reinhard et al. [3] applies a zone system that divides brightness based on human visual characteristics used in photographic techniques. The tone mapping technique of Drago et al. [4] uses adaptive logarithmic function similar to human visual perception to the image. On the other hand, a typical example of experimentally constructed algorithm is the algorithm of Schlick et al [5], and it is a technique that applies quantization to tone mapping.

In this paper, we propose an algorithm that performs tone mapping locally by dividing images into several clusters. The proposed algorithm is implemented based on HVS because it uses adaptive logarithmic mapping [4]. And because it takes into account the regional characteristics, the local contrast and detail can be improved.

The composition of this paper is as follow. Section 2 describes the conventional algorithm. The proposed algorithm is described in Section 3. Section 4 presents and analyzes the experimental results, Section 5 concludes this paper.

\section{CONVENTIONAL ALgORITHM}

\subsection{Adaptive Logarithmic Mapping}

The response of HVS about luminance is non-linear. It is approximated with equation(1) that is Weber-Fechner law.

$$
B=k_{1} \ln \left(\frac{L}{L_{0}}\right)
$$

$L_{0}$ means the luminance of background, $k_{1}$ is constant

The response is transformed to use it in image processing as equation (2)

$$
\begin{gathered}
L_{d}=\frac{\log \left(L_{w}+1\right)}{\log \left(L_{w \max }+1\right)} \\
L_{w}=\frac{L}{L_{w a}} \\
L_{w a}=\exp \left(\frac{1}{N} \sum \log [\delta+L(x, y)]\right)
\end{gathered}
$$

$L_{d}$ means displayed luminance. $L_{w}$ represents the value that input luminance is divided with $L_{w a}$. $L_{w a}$ is world adaptation that is global $\log$ average. Because $L_{w a}$ is different value corresponding to image, tone mapping function is transformed depends on image. Delta is very small value that prevent the log function to be divergence. 
Equation (2) yields the same result for all the bases, since the base of the logarithm is the same. If the base is same, it is deleted in calculation. In order to obtain different results according to the base of the $\log$, it is transformed as the following equation(5).

$$
L_{d}=\frac{\log _{b s}\left(L_{w}+1\right)}{\log _{10}\left(L_{w \max }+1\right)}
$$

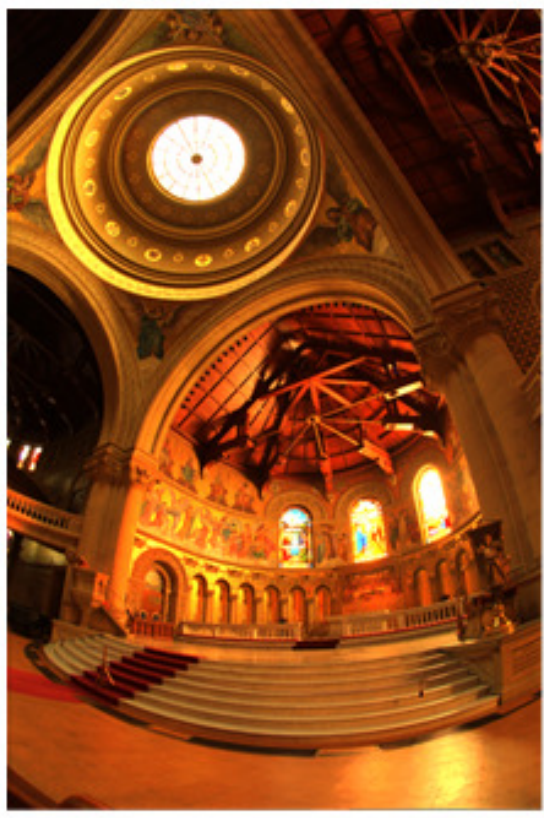

(a) $b s=2$

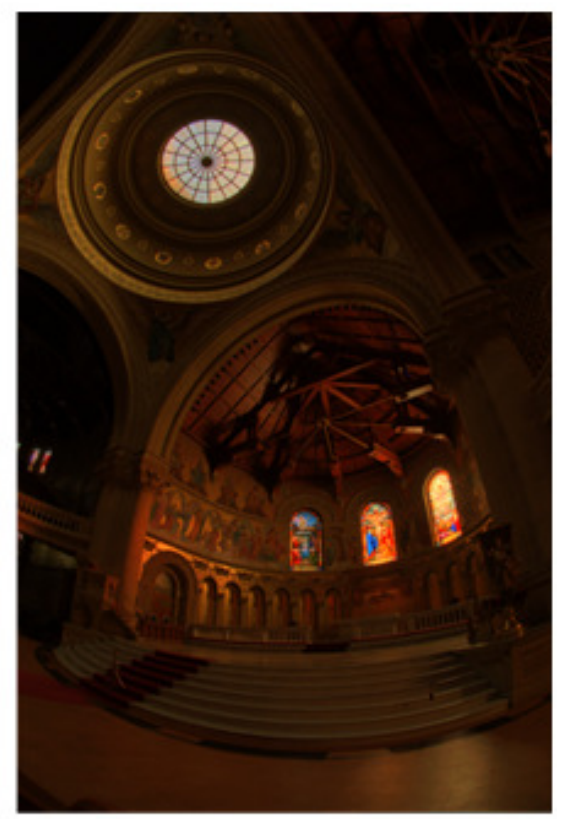

(b) $b s=10$

Figure1. experiment result according to different $b s$

If mapping is calculated based on equation (5), we use the fixed base value of $b s$. You can see the result depends on different base value in the figure 1 . In $b s=2$, the detail was well preserved and the brightness of the dark area was appropriate, and the brightness of the bright area was appropriately compressed when $b s=10$. In order to maintain two advantages, equation (6) is proposed by modifying equation (5). The output value can be calculated using Equation (6), which is a tone mapping function.

$$
L_{d}=\frac{L_{d \max } \cdot 0.01}{\log _{10}\left(L_{w \max }+1\right)} \cdot \frac{\log \left(L_{w}+1\right)}{\log \left(2+\left(\frac{L_{w}}{L_{w \max }}\right)^{\frac{\log (b)}{\log (0.5)}} \cdot 8\right)}
$$

$L_{d}, L_{w}, L_{w \max }$ is the same parameter as used in Eq. (2), and $L_{d \max }$ is the maximum displayable luminance. $b$ means user-defined constant and adjusts the degree of under-transformation. Drago et al. In [4], set the optimal $b$ to 0.85 through a survey of preference for $b$.

\subsection{Rendering HDR Image Using Integrated Global and Local Processing}

Shin et al. [6] proposed a tone mapping algorithm that integrates global and local characteristics to overcome the drawbacks of global tone mapping. This algorithm is implemented using a different tone mapping function for each pixel, and the function is obtained by considering both 
the global and the regional characteristics around each pixel. It is divided into basic global tone mapping and local tone mapping using block level averaging.

The local tone mapping is performed by referring to the characteristic of the block after forming a block of a certain size based on the current pixel. The block size should be large enough to capture local characteristics, but if it is set too large, it is difficult to determine the correct characteristics and computational complexity increases. Considering these properties, the authors determined the block size to be 3 and implemented the algorithm. To maintain overall contrast, use an average of all images mixed. The mapping function is calculated by equation (7) using mixed mean.

$$
\begin{gathered}
L_{d}=\frac{L(x, y) \cdot\left(1+\frac{\alpha L_{m b}(x, y)}{L_{\max }}\right)}{L(x, y)+C \cdot\left[(1-\alpha) L_{a v g}+\alpha L_{m b}(x, y)\right]} \\
L_{m b}=\exp \left(\frac{1}{N} \sum_{p \in \text { block }} \log \left[\delta+L_{p}\right]\right)
\end{gathered}
$$

$L_{m b}$ is the block level log mean and is defined by equation (8). $L_{\max }$ is the maximum value of the input image luminance, and $\alpha$ is a user parameter that is determined in the range of $0<\alpha<1$ and controls the ratio of the global average and the block level average. Figure 2 shows the experimental results for various $\alpha$. The authors can obtain reasonable results in the range of $0.4<\alpha<0.6$ through experiments, and assume that $\alpha=0.4$ is optimal and implement the algorithm. In the process of calculating block level averages, edge pixels have large impact on the average, resulting in halo artifacts.

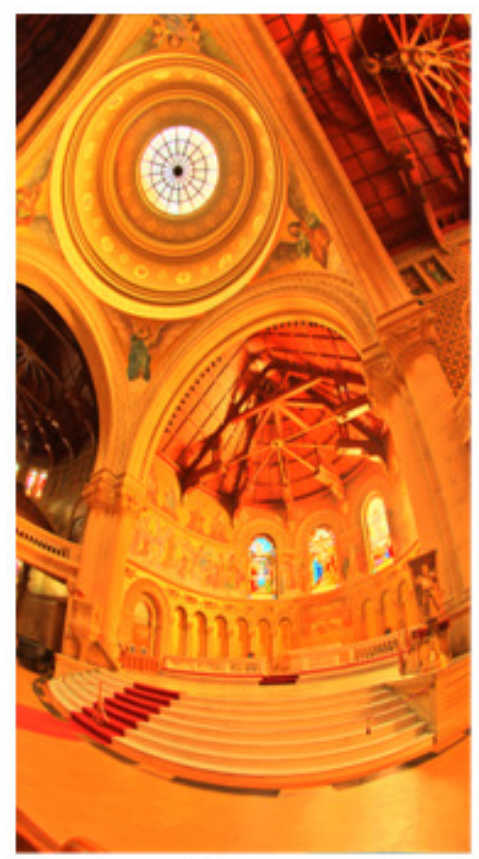

(a) $\alpha=0.2$

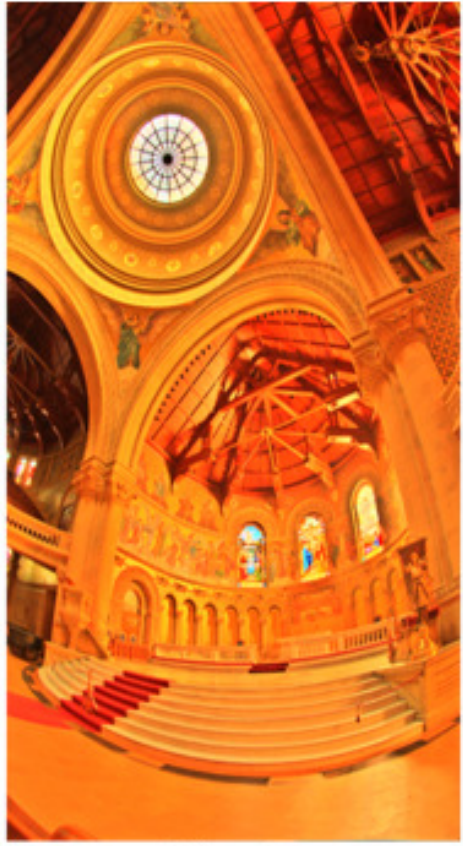

(b) $\alpha=0.4$

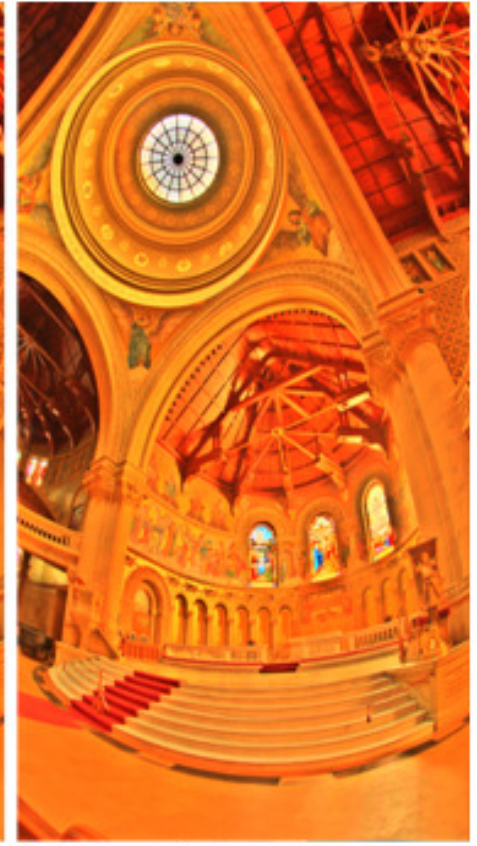

(c) $\alpha=0.6$

Figure 2. experiment result according to alpha 


\section{PROPOSED ALGORITHM}

In this paper, we propose a local tone mapping algorithm based on clustering. In Section 3.1, we introduce the clustering technique that divides the image into several clusters. In Section 3.2, local tone mapping based on clustering is interpreted to perform tone mapping according to the characteristics of each cluster. In Section 3.3, we describe a technique for eliminating boundary artifactresulting from Section 3.2.

\subsection{Clustering Algorithm}

Clustering is an algorithm that divides the image into several regions. When constructing a cluster, it is called a superpixel to bind a human visual cognition to something similar. In the proposed algorithm, the tone mapping is performed considering the regional characteristics after dividing into several regions using the SLIC (Simple Linear Iterative Clustering) technique.

The SLIC technique generates a superpixel through a process similar to k-means clustering. The SLIC technique is easy to use and can flexibly change the number of clusters to be created. The process of the SLIC begins with defining k corresponding to the size of the superpixel, which is performed in the CIELAB color space. The rest of the process is divided into two phases, initialization and assignment. In the initialization process, initialize information about the center of each cluster and the number of clusters of the corresponding pixel. Then, the assignment step is repeatedly performed to obtain an appropriately divided image.

In the initialization step, $C_{k}$ and $\operatorname{label}(i)$ are initialized. Each cluster is composed of squares with the same number of pixels, and $C_{k}$ stores information about the center of each cluster. At this time, the length of one side of the square is set to $S$. Next, the value of label( $i)$ is set to $k$ for all pixels existing in the $k$-th square.

In the assignmentstep, each information is updated to fit the image, and after a number of iterations, the information of each cluster is determined. The assignment steps follow the flowchart in figure 3. below. This step is repeated until the E corresponding to the previous result and the current result change is smaller than the specified threshold thr while repeating. Each iteration sets the surrounding pixels for the center of all the clusters. The search area of $2 S \times 2 S$ is set at the center of each cluster and the distance from center is calculated. If the calculated distance is smaller than the minimum distance $d(i)$ stored in the corresponding position, it is determined that the calculated distance is closer to the current cluster than the stored cluster, and the minimum distance $d(i)$ is newly set and the label $(i)$ of the corresponding position is changed.If we performed on all the clusters, we calculate the center for the new clusters, obtain the difference $E$ from the center of the previous clusters, and compare with $t h r$. If it is less than thr, end the iteration and complete the assignment step.

At the end of the assignment phase, the clustered images can be obtained using label information for all locations.And performs local tone mapping that improves local contrast by using clustering information. 


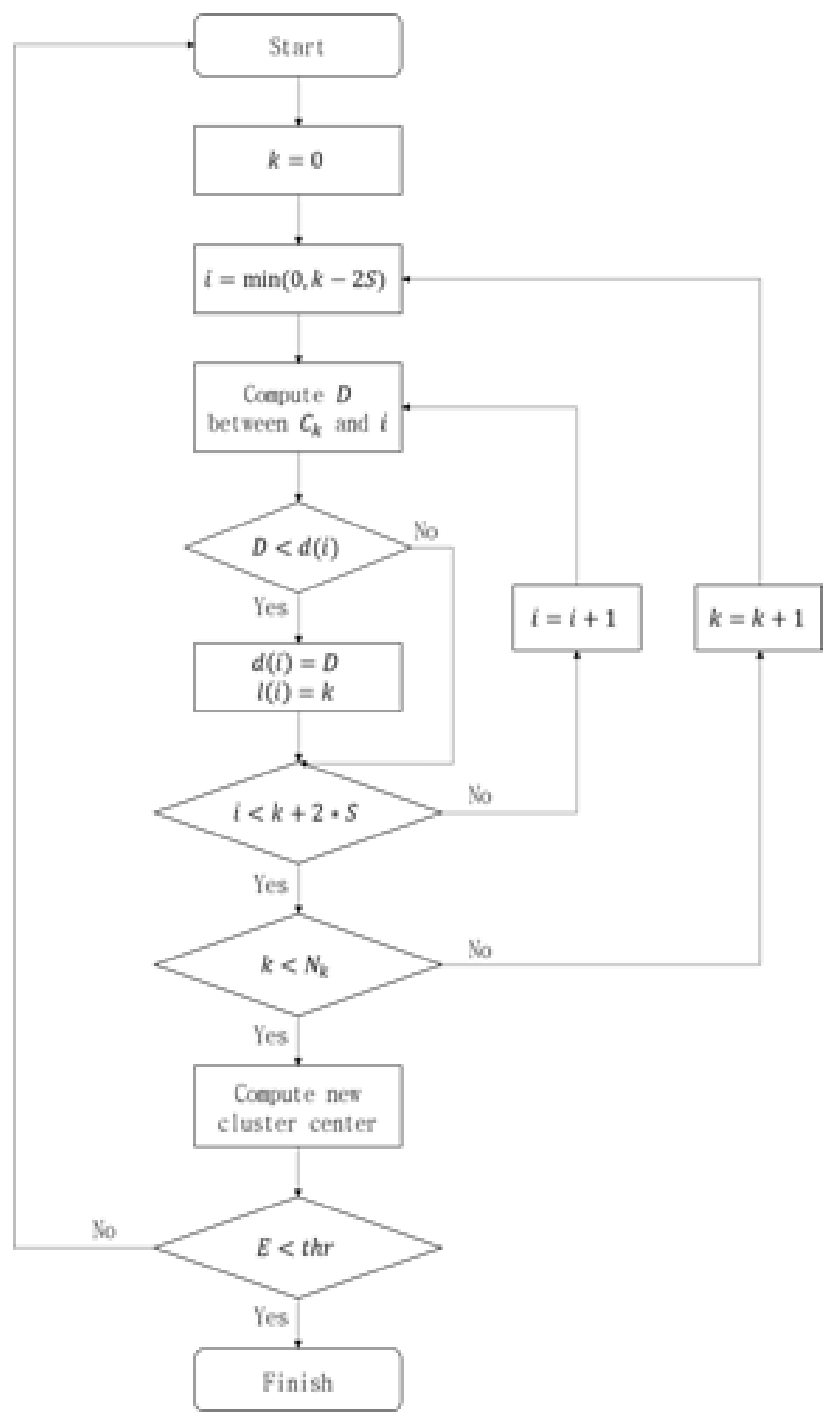

Figure 3. flow char of assignment process

\subsection{Clustering Based Local Tone Mapping}

In this paper, we propose local log mapping considering regional characteristics for each cluster in the image divided into several clusters. The proposed tone mapping scheme consists of the following flowchart. In the first step, the luminance component is extracted from the color image and the tone mapping process is performed in black and white. The tone mapping process is divided into a global process and a local process. In the global processing, the global log mean of the whole image is calculated. In the process of local processing, the image is divided into several clusters and the local log mean is obtained for each region. Prior to obtaining the local log mean, we used the SLIC technique to divide the image into several clusters that are visually cognitively similar. Find the local log mean for each subdivision. 


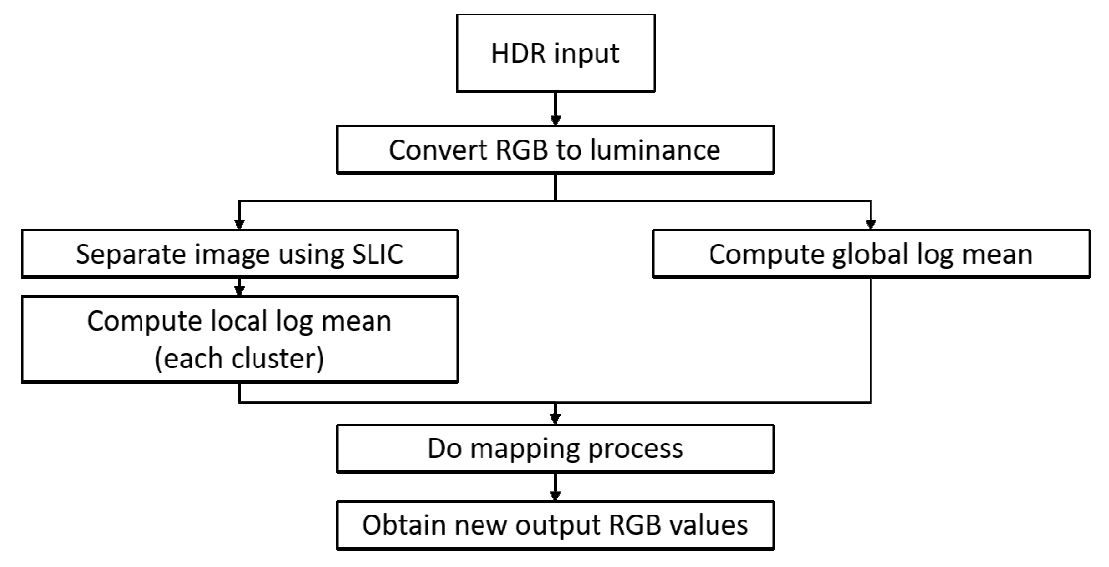

Figure 4. flow chart of proposed algorithm

The mapping process is performed after the global log average and the local log average are calculated. The mapping process is performed using different functions for each region and uses local adaptation, which is a mixture of global log average and local log average. Because local $\log$ averages differ from region to region, local adaptation also varies from region to region. When calculating the local adaptation, the global log average plays a role in maintaining overall brightness and the local log average helps to make high local contrast.

$$
\begin{gathered}
L_{l a}=(1-\alpha) L_{\text {avg }}+\alpha L_{\text {Cavg }} \\
L_{\text {Cavg }}=\exp \left(\frac{1}{N_{C}} \sum_{x, y \in \text { cluster }} \log [\delta+L(x, y)]\right)
\end{gathered}
$$

At each cluster, tone mapping process is computed using equation (11).

$$
\begin{gathered}
L_{d}=\frac{L_{d \max } \cdot 0.01}{\log _{10}\left(L_{\text {wax }}+1\right)} \cdot \frac{\log \left(L_{w}+1\right)}{\log \left(2+8 \cdot\left(\frac{L_{w}}{L_{w \max }}\right)^{c}\right)} \\
L_{w}=\frac{L}{L_{l a}}
\end{gathered}
$$

$c$ stands for user-defined constant and controls the degree to which the base is deformed. In this case, $c$ has the same role as $b$ used in the algorithm of Drago et al. [4] and has a relation of $\mathrm{c}=-\log _{2} b . \mathrm{L}_{\mathrm{w}}$ is divided by $\mathrm{L}_{\mathrm{wa}}$ in the conventional method, whereas in the proposed tone mapping technique, it is divided by the previously calculated $\mathrm{L}_{\mathrm{la}}$. This allows you to implement tone mapping that reflects local characteristics. The result of applying the proposed tone mapping technique is shown in Figure 5. 


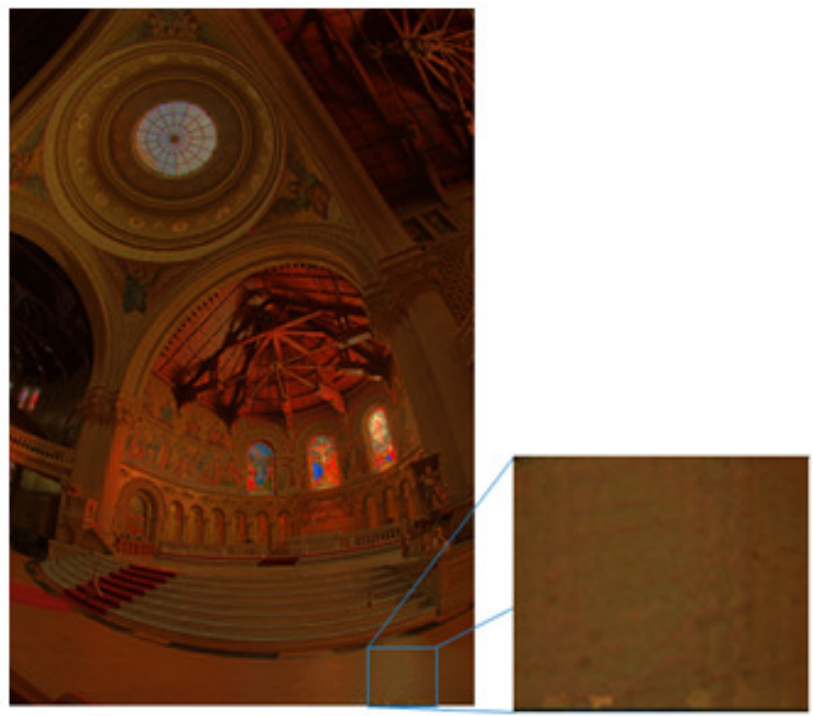

Figure5. experiment result of proposed algorithm

Experimental results show that details and local contrast are improved, but boundary artifact occur at the boundaries of the cluster. Because they are transformed using different tone mapping functions in different regions, there can be large differences in the boundaries of the clusters and clusters. In Section 3.3, we introduce techniques for removing boundary artifact.

\subsection{Dealing with Boundary Artifact}

Analysis of the previous experimental results shows that boundary artifact appear at the edge of the cluster. When performing the tone mapping process to remove boundary artifact, the tone mapping function of surrounding clusters is considered. We use the boundary artifact removal technique introduced in the algorithm of Duan et al [7].

This technique is performed as shown in Fig. 6. Using the tone mapping functions of surrounding clusters, the current pixel is input to each function and the weighted sum of the output result is mapped. The weight for each result is measured in inverse proportion to the distance from the current pixel, and the distance is calculated by Euclidean distance between the center of the cluster and the current pixel, and the weight is calculated by equation (13). If we calculate each weight, we get the tone-mapped result through equation (14). All pixels undergo such a transformation that results in the elimination of borderline defects.

$$
\begin{gathered}
w_{d}=\exp \left(-\frac{d_{n}}{\sigma_{n}}\right) \\
d_{n}=\frac{\sum T M O(D(x, y)) \cdot w_{d}(n)}{\sum w_{d}(n)}
\end{gathered}
$$




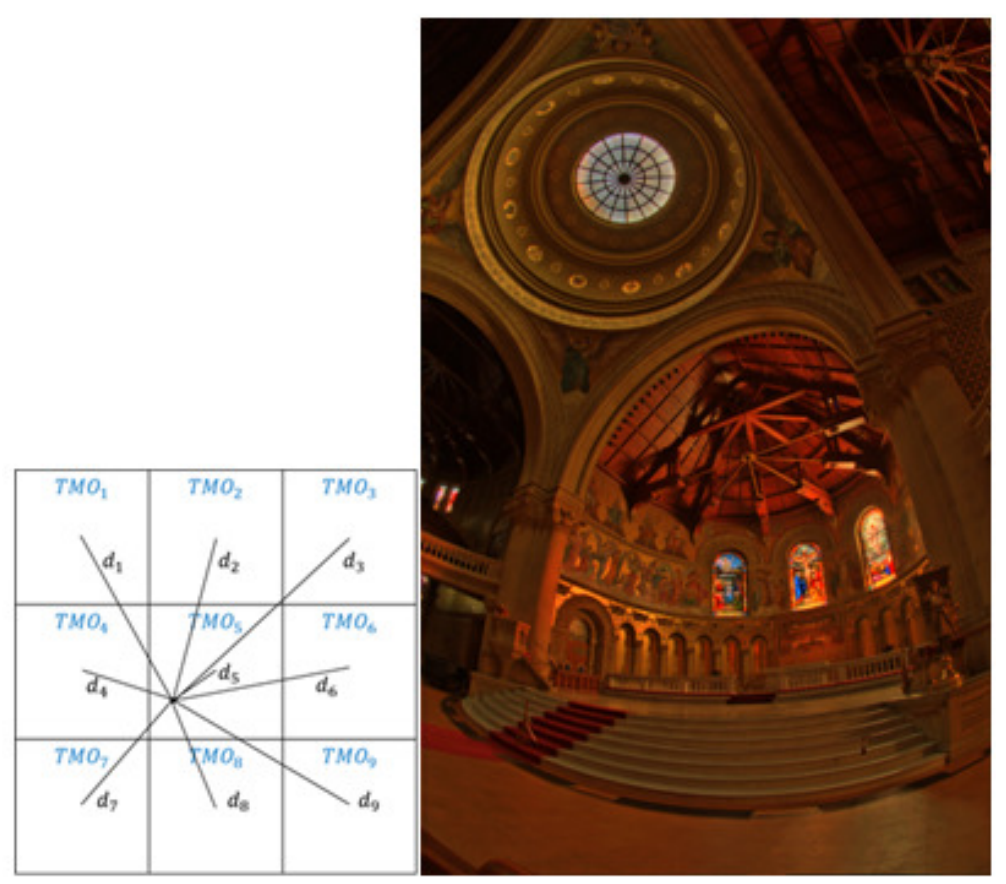

Figure 6. removing boundary artifact. (a) example of calculating weight, (b) experiment result removed boundary artifact.

\section{EXPERIMENT RESULT}

In this section, we compare and analyze the experiment results to compare the proposed tone mapping algorithm with the conventional tone mapping algorithm.All image processing is performed via MATLAB 2015a in a PC environment of Windows 10 64-bit operating system Intel Core i5-3470 CPU @ 3.20GHz. In HDR images, there is no objective image quality index commonly used such as PSNR (Peak Signal to Noise Ratio), which is mainly used for image quality comparison of general images. Therefore, it is necessary to rely on subjective image quality comparison. We evaluate the performance of the proposed tone mapping technique through subjective image quality comparison.

Figure 7 compares the experimental results of Drago et al. [4] and the proposed tone mapping algorithm for KitchenWindow.hdr image. The proposed tone mapping preserves more of the local contrast or details. At (a), it can be seen that the overall brightness of the image is uniformly distributed, and the dynamic range is appropriately compressed. However, you can see the shadows in the dark areas. And when you look at the part of the window, it is too bright to know exactly what kind of landscape it is.The result of the proposed tone mapping of (b) shows the result of complementing the disadvantage of (a) mentioned above. First, we got the result of utilizing the detail of the dark part. The letter in the box on the right shelf can be seen more clearly compared to (a). You can also recognize the coffee machine or the stuffs next to it. Finally, when you look at the image on the window side, you can see in detail what kind of scenery is out of the window and color information can be expressed more abundantly.

Figure 8 compares the experimental results for memorial.hdr images. This image has a very bright area of stained glass and a window in the ceiling, and a very dark area in the corner of the ceiling. The results of (a) show that the overall brightness is distributed evenly, but the details are not preserved in very bright or dark areas. Especially, the stained glass on rightmost side can not recognize any picture. Looking at (b), the details were preserved in the corner areas of the ceiling, which is a dark area, and many details were preserved in the stained glass area. The picture of 
rightmost stained glass which was not seen in (a) is recognizable. Comparing to (a), it can be seen that more information is expressed.

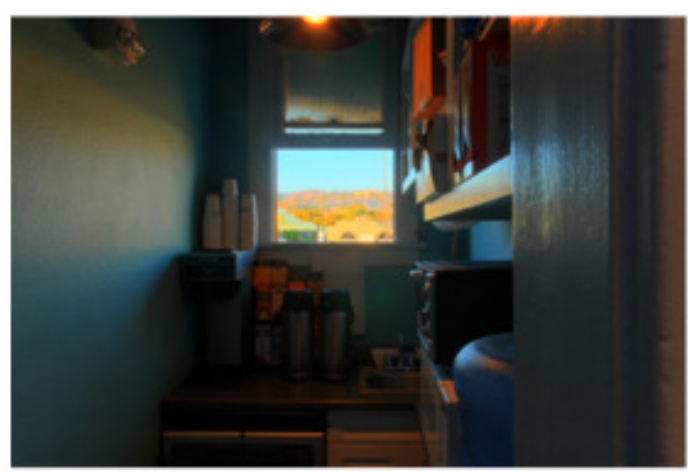

(a)

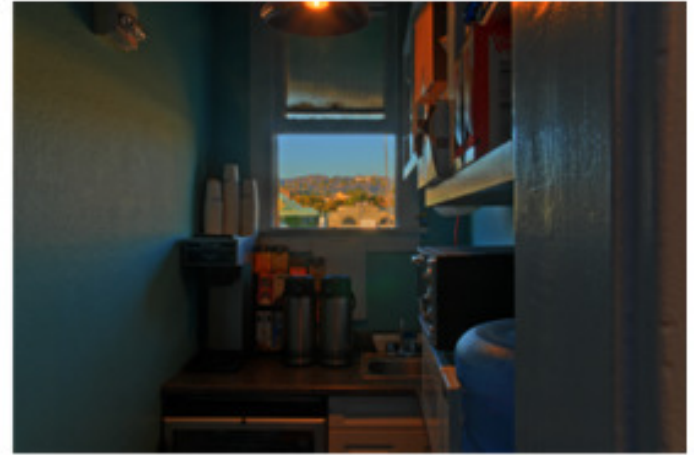

(b)

Figure 7. subjective quality comparison, (a): the result of Drago et al. [4], (b): the result of proposed algorithm

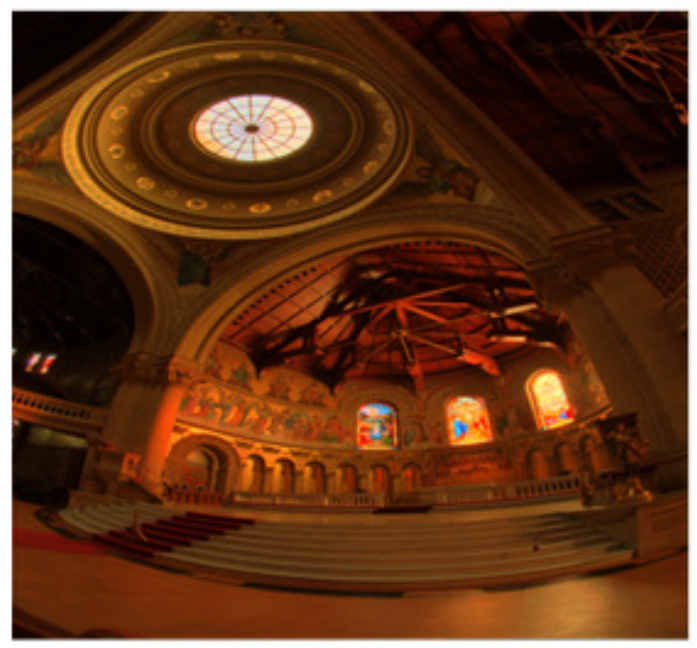

(a)

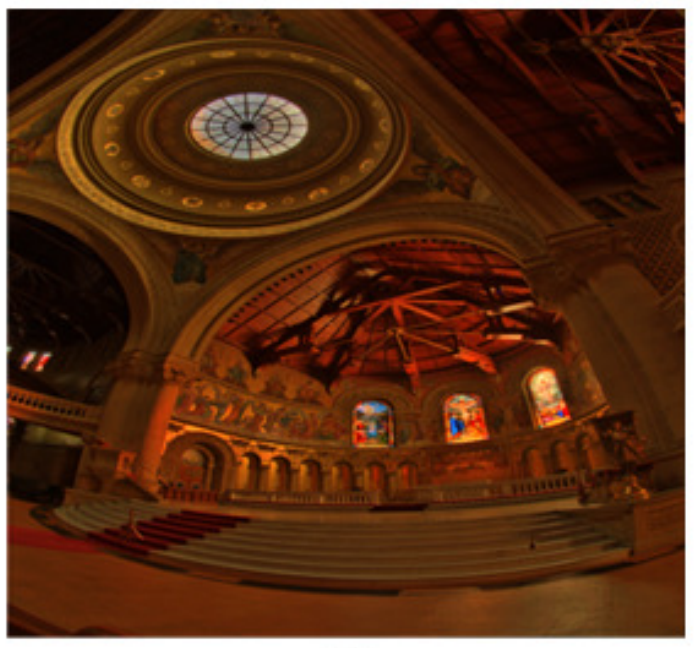

(b)

Figure 8. subjective quality comparison

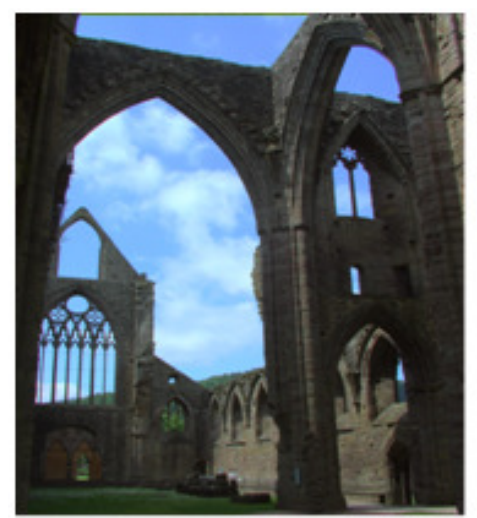

(a)

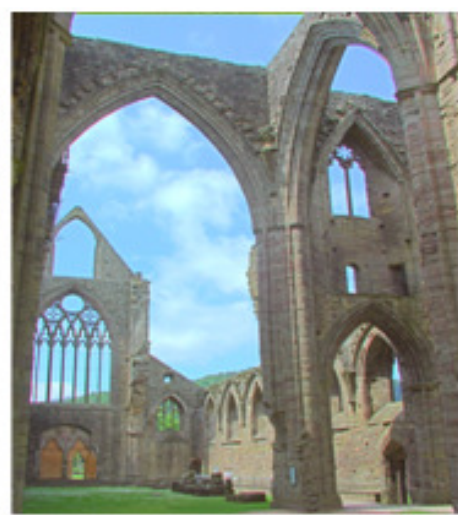

(b)

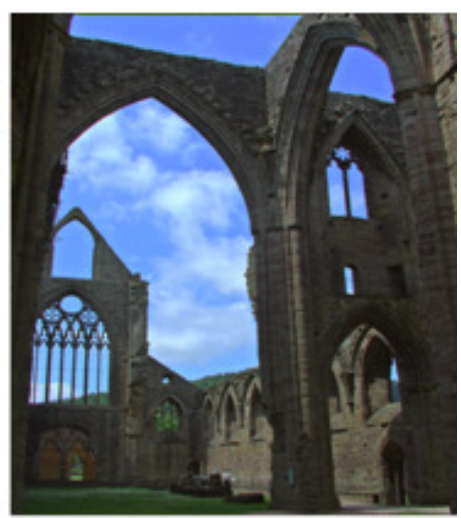

(c)

Figure 9. subjective quality comparison 


\section{CONCLUSION}

In this paper, we propose a tone mapping algorithm considering local characteristics after divides image with several regions based on clustering. In order to identify local characteristics, cluster level $\log$ mean was calculated for each cluster, and local adaptation was performed by mixing global log mean and local log mean of each cluster, and tone mapping was performed using local adaptation. In order to maintain the visual quality in the tone mapping process, the result image is configured to have a brightness distribution similar to that of human visual system using adaptive logarithmic mapping based on the visual recognition. Experiments have shown that visual quality is maintained similar to adaptive log mapping based on visual cognition, that local contrast is high and local detail is improved. And we show that the proposed tone mapping has superior performance compared with conventional tone mapping algorithm.

\section{ACKNOWLEDGEMENTS}

The authors would like to thank everyone, just everyone!

\section{REFERENCES}

[1] R. Boitard, M. Pourazad, P. Nasiopoulos, and J. Slevinsky, (2015) "Demystifying High-DynamicRange Technology: A new Evolution in Digital Media," IEEE Consum. Electron. Mag., Vol. 4, No. 4, pp. 72-86.

[2] C. Ha, J. Lee, and J. Jeong, (2016) "Tone Reproduction for High-Dynamic Range Imaging based on Adaptive Filtering,” Opt. Eng., Vol. 53, No.3, p.33103.

[3] E. Reinhard, M. Stark, P. Shirley, and J. Ferwerda, (2002) "Photographic Tone Reproduction for Digital Images," ACM Trans. Graph., Vol. 21, No. 3, pp. 267-276.

[4] F. Drago, K.Myszkowski, T. Annen, and N. Chiba, (2003) "Adaptive Logarithmic Mapping for Displaying High Contrast Scenes," Comput. Graph. Forum, Vol. 22, No. 3, pp. 419-426.

[5] C. Schlick, (1994) "Quantization Techniques for Visualization of High Dynamic Range Pictures," Proceeding of the Fifth Eurogrphics Workshop on Rendering, pp. 7-18.

[6] H. Shin, T. Yu, Y. Ismail, and B. Saeed, (2011) "Rendering High Dynamic Range Images by using Integrated Global and Local Processing,” Opt. Eng., Vol. 50, No.11, p.117002.

[7] J. Duan, M. Bressan, C. Dance, and G. Qiu, (2010) "Tone-mapping High Dynamic Range Images by Novel Histogram Adjustment,” Pattern Recognition, Vol. 43, No. 5, pp. 1847-1962.

[8] K. Lee, W. Choe, J. Kwon, and S. Lee, (2009) "Locally Adaptive High Dynamic Range Image Reproduction Inspired by Human Visual System,” Proc. SPIE 7241, pp. 72410T 